\title{
Booster vaccines protect hamsters with waning immunity from Delta VOC infection, disease, and transmission
}

Kyle G. Potts ${ }^{1,2,3} \dagger$, Ryan S. Noyce ${ }^{4,5} \dagger$, Chris Gafuik ${ }^{1,2,3}$, Cini M. John ${ }^{1,2,3}$, Hayley M. Todesco $^{1,2,3}$, Elaine De Heuvel ${ }^{3,6,7}$, Nicole Favis ${ }^{4,5}$, Margaret M. Kelly ${ }^{3,6,7}$, David H. Evans ${ }^{4,5^{*}}$, Douglas J. Mahoney ${ }^{1,2,3,8,9 *}$

${ }^{1}$ Arnie Charbonneau Cancer Institute, Faculty of Medicine, University of Calgary; Calgary, Alberta, Canada.

${ }^{2}$ Alberta Children's Hospital Research Institute, Faculty of Medicine, University of Calgary; Calgary, Alberta, Canada.

${ }^{3}$ Snyder Institute for Chronic Disease, Faculty of Medicine, University of Calgary; Calgary, Alberta, Canada.

${ }^{4}$ Department of Medical Microbiology and Immunology, Faculty of Medicine and Dentistry, University of Alberta; Edmonton, Alberta, Canada.

Edmonton, Alberta, Canada.

${ }^{6}$ Department of Pathology and Laboratory Medicine, Faculty of Medicine, University of Calgary; Calgary, Alberta, Canada.

${ }^{7}$ Department of Physiology and Pharmacology, Faculty of Medicine, University of Calgary; Calgary, Alberta, Canada. 


\title{
Template revised February 2021
}

${ }^{8}$ Department of Microbiology, Immunology and Infectious Disease, Faculty of Medicine, University of Calgary; Calgary, Alberta, Canada.

${ }^{9}$ Department of Biochemistry and Molecular Biology, Faculty of Medicine, University of Calgary; Calgary, Alberta, Canada.

*Corresponding author. Email: djmahone@ucalgary.ca; devans@ualberta.ca

$\dagger$ These authors contributed equally to this work

\begin{abstract}
Waning immunity to COVID-19 vaccination is associated with increased risk of breakthrough infection, especially with highly transmissible variants of concern (VOC). Booster vaccination generates rapid immune recall in humans, which real-world observational studies suggest protects against VOC infection and associated disease, and modeling studies suggest could mitigate community spread. We directly tested the impact of booster vaccination on protection against Delta VOC infection, disease, and transmission to naïve cohorts in golden Syrian hamsters. Animals with waning immunity to bnt162b2 generated rapid immune recall and 15 strong protection against upper- and lower-respiratory tract infection when boosted with bnt126b2, mRNA-1273 or AZD1222. Boosting with either mRNA vaccine generated moderate protection against lung inflammation and virus transmission to unvaccinated animals. Our data support booster vaccination as a tool to address emerging VOC in the COVID-19 pandemic.
\end{abstract} vaccination protects hamsters from Delta VOC infection, disease, and transmission. 


\section{Template revised February 2021}

Main Text: Accumulating real-world evidence demonstrates that immunity to SARS-CoV-2 wanes significantly within 3-8 months of vaccination ${ }^{1-4}$. Waning immunity is associated with decreased vaccine efficacy, especially against infection and mild disease, but also (although to a lesser extent) against severe disease ${ }^{5-9}$. Breakthrough infection is most common with highly transmissible VOC such as Delta and Omicron, which replicate quickly, partially evade antibodies elicited by vaccination against the ancestral strain, and represent most SARS-CoV-2 infections worldwide ${ }^{10-16}$. Third dose boosting campaigns have therefore been initiated in many countries, with recent observational studies demonstrating a rapid increase in serum neutralizing antibody (NAb) titer and breadth against highly transmissible VOC concomitant with a decrease in infection rate and associated disease ${ }^{17-26}$. Modeling studies have also suggested that booster vaccination could reduce community transmission ${ }^{27}$. While these data are encouraging, the impact of booster vaccination after waning immunity has not been determined in a rigorously controlled experimental setting. Studies in preclinical models would be highly complementary to real-world data, as they allow for control over important variables such as the duration, dose, and timing of virus exposure as well as behaviors that could influence infection susceptibility. They also enable direct assessment of virus infection and associated pathology in the lung, which should provide insight into booster vaccination effectiveness beyond its impact on disease symptoms.

We therefore conducted a Delta VOC challenge-transmission study in golden Syrian hamsters immunized for 9 months with bnt162b2 and then boosted with a third dose of either bnt162b2, mRNA-1273 or AZD1222 (Fig. 1). As expected, naïve hamsters vaccinated with two doses of bnt $162 \mathrm{~b} 2$ separated by 21 days generated a robust serum NAb response against SARS-CoV-2, as measured by pseudovirus PRNT 50 assay (Fig. 2a). Mean serum NAb titers peaked on day 35 to 


\section{Template revised February 2021}

levels similar to those measured in humans 35 days after an identical vaccination regimen (Fig. 2b). Once peaked, serum NAb titers waned progressively until becoming undetectable on day 250 (Fig. 2a). These data confirm waning immunity to mRNA vaccination against SARS-CoV-2 in the golden Syrian hamster, as was shown recently after natural infection ${ }^{28,29}$. The similarity to which serum NAb titers were generated and then waned relative to humans provides support for the hamster as a high-fidelity model for studying booster vaccination ${ }^{30,31}$.

On day 275, hamsters were boosted with 5ug bnt162b2, 15 ug mRNA-1273 or 8e9 particles AZD1222, which are doses normalized to the clinical dosing regimen in humans. Booster vaccination was well tolerated and generated rapid serum NAb recall against SARS-CoV-2 (Fig. 2c, d). Serum NAb titers increased by 3 days and reached mean $\mathrm{NT}_{50}$ of $435 \pm 223,234 \pm 994$ and $138 \pm 108$ for bnt162b2, mRNA-1273, and AZD1222, respectively, at 10 days. The kinetics and strength of $\mathrm{NT}_{50}$ recall are similar to those reported in humans boosted 3-8 months after primary mRNA vaccination ${ }^{17-19}$ and above serum NAb titers determined to be protective in humans ${ }^{32}$.

Fifteen days after booster vaccination, hamsters were challenged intranasally with 1e5 plaque forming units (pfu) Delta VOC. Unvaccinated hamsters lost weight post-challenge (Fig. 3a) and generated modest serum NAb titers against authentic Delta VOC by day 4 (Fig. 3b). These animals supported high infectious viral titers in the upper (nasal swab on day 2 and 4, Fig. 3c) and lower (lung on day 4, Fig. 3d) respiratory tract and prominent N-reactivity in alveoli and bronchial epithelium on day 4 (Fig. 3e-g). They also had pronounced inflammation in the lung, characterized by variable peribronchial, perivascular and peripheral inflammation involving the alveolar septae and spaces (consisting mainly of mononuclear cells), prominent neutrophilic and eosinophilic infiltrates with involvement of the vascular wall, and focal fibrosis within the small 


\section{Template revised February 2021}

airways (Fig 4a, b). These data are consistent with previous reports demonstrating that golden Syrian hamsters are highly susceptibility to SARS-CoV-2 infection and associated inflammation in the lung ${ }^{33,34}$. weight after challenge (Fig. 3a) and generated a stronger anamnestic serum NAb response to authentic Delta VOC (Fig. 3b). These animals supported similar infectious viral titers in URT on day 2 but less on day 4 (Fig. 3c), consistent with human studies reporting similar peak viral load in URT but accelerated viral clearance in breakthrough compared to primary infection with Delta VOC $^{35,36}$. In the lungs, fewer infectious particles (Fig. 3d) and N-reactive cells (Fig. 3e-g) were detected in hamsters with waning immunity, as was reported in monkeys challenged with Delta VOC one year after vaccination with mRNA-1273 ${ }^{37}$. However, a similar degree of lung inflammation was observed (Fig. 4a, b). Collectively, these data show that hamsters with waning immunity to bnt162b2 are highly susceptible to URT infection by Delta VOC but clear it faster than unvaccinated animals and are modestly- to highly susceptible to infection and associated inflammation in the lung, respectively. A qualitatively similar response was recently reported in hamsters with waning immunity to natural infection ${ }^{28,29}$.

Hamsters delivered a booster vaccine lost similar weight to non-boosted animals by day 2 postchallenge but began to recover their weight by day 4 (Fig. 3a). These animals generated a strong anamnestic serum NAb response to authentic Delta VOC (Fig. 3b), supported fewer infectious viral titers in URT on day 2 and day 4 (Fig. 3c), supported no detectable infectious viral titers in lung (Fig. 3d), and harbored fewer cells with N-reactivity in the lung (Fig. 3e-g). There were no differences between animals boosted with bnt162b2, mRNA1273 or AZD1222 in any of these 


\section{Template revised February 2021}

measures. However, the impact of booster vaccination on lung inflammation after Delta VOC challenge diverged between groups, where a third dose of bnt162b2 was most protective, followed by mRNA-1273 and then AZD1222, which generated no measurable protection on day 4 (Fig. 4a, b). Taken together, these data are consistent with recent reports that bnt162b2, mRNA-1273 and adenovirus-vectored booster vaccines generate rapid and functional immune recall in humans with waning immunity to a primary bnt162b2 vaccination ${ }^{17-23}$. They also raise the prospect that third dose bnt $162 \mathrm{~b} 2$ vaccination after waning immunity to bnt $162 \mathrm{~b} 2$ offers superior protection against lung inflammation than either mRNA-1273 or AZD1222. immunity from spreading Delta VOC to unvaccinated animals. Twenty-four hours after challenge, hamsters were co-housed 1:1 with naive hamsters. Co-housed animals were separated by a porous aluminum barrier and then moved into individual cages after 24 hours. Nasal swabs were taken 48 hours after cohousing. As expected, naïve hamsters co-housed with unvaccinatedchallenged hamsters were robustly infected by Delta VOC (Fig. 4c), to even higher levels than challenged animals (Fig. 3c). Naïve hamsters co-housed with animals with waning immunity had 3-fold lower infectious viral titers in nasal swabs at 48 hours (Fig. 4c). In contrast, naïve hamsters co-housed with animals boosted with bnt162b2 or mRNA-1273 (but not AZD1222) had $>10$-fold lower viral titers (Fig. 4c). These data show that hamsters with waning immunity are partly protected from transmitting Delta VOC to unvaccinated animals, and that boosting with bnt162b2 or mRNA-1273 generates further protection in these experimental conditions.

Our results complement real-world studies in humans showing waning immunity after primary vaccination concomitant with decreased protection against VOC infection and disease ${ }^{1-8}$. Also 


\section{Template revised February 2021}

complementary is the rapid immune recall generated by booster vaccination that is associated with increased protection against VOC infection and disease ${ }^{17-23}$. We add to these observations direct evidence that booster vaccination reduces Delta VOC infectious viral load in the URT and lung and generates measurable protection against lung inflammation and viral transmission to unvaccinated animals. The protection against URT infection and transmission is noteworthy, as bnt162b2, mRNA-1273 and AZD1222 were not designed to generate mucosal immunity, but rather protect against severe disease. A decrease in URT viral load post-breakthrough infection with Delta VOC after bnt $162 \mathrm{~b} 2$ booster vaccination was also reported in humans ${ }^{38}$. Whether this would hold for even more transmissible VOC such as Omicron remains unknown, but even a modest decrease in URT load and viral transmission could have a significant beneficial impact on community spread. It is also noteworthy that animals boosted with AZD1222 were protected against URT and lung infection but not lung inflammation or viral transmission. This is consistent with a recent observational study showing that bnt $162 \mathrm{~b} 2$ vaccination protected against transmission better than ADZ1222 ${ }^{39}$. One explanation is that AZD1222 was less effective at boosting cellular immunity, which could have changed the inflammatory response in the lung. As transmission is influenced by both infectious viral load and animal behavior (e.g., breathing rate, coughing, sneezing, etc.), the elevated lung inflammation may have altered animal behavior in ways that facilitated viral spread even though viral load was similar.

Our study has several limitations. First, as direct intranasal challenge forces a breakthrough infection, our study was designed to evaluate the impact of booster vaccination on the severity of breakthrough infection but not its incidence, which observational studies have shown is reduced by booster vaccination ${ }^{20}$. Similarly, cohousing animals for 24 hours at peak URT infection is an extreme model of transmission. Together these factors lead us to speculate that our study 


\section{Template revised February 2021}

underestimates the overall beneficial impact of booster vaccination in the real world. Second, the size of each experimental group was small. This substantially impacted our statistical power, especially when the effect size was small (e.g., transmission, Fig. 4c) or the biological variability was high (e.g., anamnestic response, Fig. 3b). As such, our interpretations are sometimes based on non-significant data trends. Third, there are few validated antibody reagents for hamster studies, which limited our ability to probe cellular immunity or assess lung inflammation in depth. The cellular infiltration measured on H\&E likely represents both pathological and physiological inflammation. Moreover, our assessment on day 4 only captures a snapshot of the inflammatory response to Delta VOC. Fourth, our study did not track the kinetics or durability of immune response beyond 10 days post-boost. A recent study in humans showed that bnt162b2boosted immunity peaked within 2 weeks whereas adenovirus-vectored vaccine (Ad26)-boosted immunity continued to rise until at least 1 month ${ }^{19}$. The differences we observed between the mRNA vaccines and AZD1222 may therefore have resulted from the timing of Delta VOC challenge after boost.

We conclude that booster immunization with clinically relevant COVID-19 vaccines generates rapid protection against Delta VOC challenge in animals with waning immunity. However, because protection was incomplete and highly transmissible VOC such as Omicron continue to emerge ${ }^{40}$, our study supports the development of more effective booster vaccines, potentially including intranasally-delivered, variant-specific and/or multi-valent formulations, and indicate that non-pharmaceutic interventions may still be required to curb infection and disease as the pandemic evolves. 


\section{Template revised February 2021}

\section{References:}

1. Canaday, D. H. et al. Significant reduction in humoral immunity among healthcare workers and nursing home residents 6 months after COVID-19 BNT162b2 mRNA vaccination. medRxiv 2021.08.15.21262067 (2021). doi:10.1101/2021.08.15.21262067

2. Levin, E. G. et al. Waning Immune Humoral Response to BNT162b2 Covid-19 Vaccine over 6 Months. N. Engl. J. Med. 385, e84 (2021).

3. Baden, L. R. et al. Phase 3 Trial of mRNA-1273 during the Delta-Variant Surge. https://doi.org/10.1056/NEJMc2115597 (2021). doi:10.1056/NEJMC2115597

4. Collier, A. Y. et al. Differential Kinetics of Immune Responses Elicited by Covid-19 Vaccines. N. Engl. J. Med. 385, 2010-2012 (2021).

5. Bruxvoort, K. J. et al. Effectiveness of mRNA-1273 against delta, mu, and other emerging variants of SARS-CoV-2: test negative case-control study. doi:10.1136/bmj-2021-068848

6. Rosenberg, E. S. et al. Covid-19 Vaccine Effectiveness in New York State. https://doi.org/10.1056/NEJMoa2116063 (2021). doi:10.1056/NEJMOA2116063

7. Goldberg, Y. et al. Waning Immunity after the BNT162b2 Vaccine in Israel. https://doi.org/10.1056/NEJMoa2114228 (2021). doi:10.1056/NEJMOA2114228

8. Thomas, S. J. et al. Safety and Efficacy of the BNT162b2 mRNA Covid-19 Vaccine through 6 Months. N. Engl. J. Med. 385, 1761-1773 (2021).

9. Katikireddi, S. V. et al. Two-dose ChAdOx1 nCoV-19 vaccine protection against COVID-19 hospital admissions and deaths over time: a retrospective, population-based cohort study in Scotland and Brazil. Lancet (London, England) (2021). doi:10.1016/S0140-6736(21)02754-9

10. Roessler, A., Riepler, L., Bante, D., Laer, D. von \& Kimpel, J. SARS-CoV-2 B.1.1.529 variant (Omicron) evades neutralization by sera from vaccinated and convalescent 


\section{Template revised February 2021}

individuals. medRxiv 2021.12.08.21267491 (2021). doi:10.1101/2021.12.08.21267491

11. Andrews, N. et al. Effectiveness of COVID-19 vaccines against the Omicron (B.1.1.529) variant of concern.

12. Pulliam, J. R. C. et al. Increased risk of SARS-CoV-2 reinfection associated with emergence of the Omicron variant in South Africa. doi:10.1101/2021.11.11.21266068

13. Siddle, K. J. et al. Transmission from vaccinated individuals in a large SARS-CoV-2 Delta variant outbreak. Cell 0, (2021).

14. Planas, D. et al. Considerable escape of SARS-CoV-2 Omicron to antibody neutralization. Nature (2021). doi:10.1038/D41586-021-03827-2

15. Liu, L. et al. Striking antibody evasion manifested by the Omicron variant of SARS-CoV2. Nature (2021). doi:10.1038/D41586-021-03826-3

16. Cameroni, E. et al. Broadly neutralizing antibodies overcome SARS-CoV-2 Omicron antigenic shift. Nature (2021). doi:10.1038/D41586-021-03825-4

17. Falsey, A. R. et al. SARS-CoV-2 Neutralization with BNT162b2 Vaccine Dose 3. N. Engl. J. Med. 385, 1627-1629 (2021).

18. Atmar, R. L. et al. Heterologous SARS-CoV-2 Booster Vaccinations - Preliminary Report. medRxiv 2021.10.10.21264827 (2021). doi:10.1101/2021.10.10.21264827

19. Tan, C. S. et al. Ad26.COV2.S or BNT162b2 Boosting of BNT162b2 Vaccinated Individuals. medRxiv 2021.12.02.21267198 (2021). doi:10.1101/2021.12.02.21267198

20. Bar-On, Y. M. et al. Protection of BNT162b2 Vaccine Booster against Covid-19 in Israel. N. Engl. J. Med. 385, 1393-1400 (2021).

21. Barda, N. et al. Effectiveness of a third dose of the BNT162b2 mRNA COVID-19 vaccine for preventing severe outcomes in Israel: an observational study. Lancet 398, 2093-2100 (2021). 


\section{Template revised February 2021}

22. Munro, A. P. S. et al. Safety and immunogenicity of seven COVID-19 vaccines as a third dose (booster) following two doses of ChAdOx1 nCov-19 or BNT162b2 in the UK (COVBOOST): a blinded, multicentre, randomised, controlled, phase 2 trial. Lancet $\mathbf{0},(2021)$.

23. Basile, K. et al. Improved neutralization of the SARS-CoV-2 Omicron variant after

Pfizer-BioNTech BNT162b2 COVID-19 vaccine boosting. bioRxiv 2021.12.12.472252 (2021). doi:10.1101/2021.12.12.472252

24. Muhsen, K. et al. Effects of BNT162b2 Covid-19 Vaccine Booster in Long-Term Care Facilities in Israel. https://doi.org/10.1056/NEJMc2117385 (2021). doi:10.1056/NEJMC2117385

25. Arbel, R. et al. BNT162b2 Vaccine Booster and Mortality Due to Covid-19. https://doi.org/10.1056/NEJMoa2115624 385, 2413-2420 (2021).

26. Bar-On, Y. M. et al. Protection against Covid-19 by BNT162b2 Booster across Age Groups. N. Engl. J. Med. 385, 2421-2451 (2021).

27. Gardner, B. J. \& Kilpatrick, A. M. Third doses of COVID-19 vaccines reduce infection and transmission of SARS-CoV-2 and could prevent future surges in some populations: a modeling study. medRxiv 2021.10.25.21265500 (2021). doi:10.1101/2021.10.25.21265500

28. Mohandas, S. et al. Protective immunity of the primary SARS-CoV-2 infection reduces disease severity post re-infection with Delta variants in Syrian hamsters. bioRxiv 2021.11.28.470293 (2021). doi:10.1101/2021.11.28.470293

29. Horiuchi, S. et al. Immune memory from SARS-CoV-2 infection in hamsters provides variant-independent protection but still allows virus transmission. Sci. Immunol. eabm3131 (2021). doi:10.1126/SCIIMMUNOL.ABM3131/SUPPL_FILE/SCIIMMUNOL.ABM3131_TABL 


\section{Template revised February 2021}

\section{E_S1.ZIP}

30. Pegu, A. et al. Durability of mRNA-1273 vaccine-induced antibodies against SARS-

CoV-2 variants. Science (80-. ). eabj4176 (2021). doi:10.1126/SCIENCE.ABJ4176

31. Suthar, M. S. et al. Durability of immune responses to the BNT162b2 mRNA vaccine.

bioRxiv 2021.09.30.462488 (2021). doi:10.1101/2021.09.30.462488

32. Gilbert, P. B. et al. Immune correlates analysis of the mRNA-1273 COVID-19 vaccine efficacy clinical trial. Science eab3435 (2021).

33. Rosenke, K. et al. Defining the Syrian hamster as a highly susceptible preclinical model for SARS-CoV-2 infection. Emerg. Microbes Infect. 9, 2673 (2020).

34. Sia, S. F. et al. Pathogenesis and transmission of SARS-CoV-2 in golden hamsters. Nature (2020). doi:10.1038/s41586-020-2342-5

35. Singanayagam, A. et al. Community transmission and viral load kinetics of the SARSCoV-2 delta (B.1.617.2) variant in vaccinated and unvaccinated individuals in the UK: a prospective, longitudinal, cohort study. Lancet Infect. Dis. 0, (2021).

36. Chia, P. Y. et al. Virological and serological kinetics of SARS-CoV-2 Delta variant vaccine-breakthrough infections: a multi-center cohort study. medRxiv 2021.07.28.21261295 (2021). doi:10.1101/2021.07.28.21261295

37. Gagne, M. et al. Protection from SARS-CoV-2 Delta one year after mRNA-1273 vaccination in rhesus macaques is coincident with anamnestic antibody response in the lung. Cell 0, (2021).

38. Levine-Tiefenbrun, M. et al. Viral loads of Delta-variant SARS-CoV2 breakthrough infections following vaccination and booster with the BNT162b2 vaccine. medRxiv 2021.08.29.21262798 (2021). doi:10.1101/2021.08.29.21262798

39. Eyre, D. W. et al. The impact of SARS-CoV-2 vaccination on Alpha \&amp; Delta variant 
transmission. medRxiv 2021.09.28.21264260 (2021). doi:10.1101/2021.09.28.21264260

40. Callaway, E. Beyond Omicron: what's next for COVID's viral evolution. Nature 600, 204-207 (2021).

41. Cimolai, N., Taylor, G. P., Mah, D. \& Morrison, B. J. Definition and Application of a Histopathological Scoring Scheme for an Animal Model of Acute Mycoplasma pneumoniae Pulmonary Infection. Microbiol. Immunol. 36, 465-478 (1992).

42. Lammers, A. J. J., de Porto, A. P. N. A., de Boer, O. J., Florquin, S. \& van der Poll, T. The role of TLR2 in the host response to pneumococcal pneumonia in absence of the spleen. BMC Infect. Dis. 12, 1-9 (2012).

43. Voynow, J. A. et al. Neutrophil elastase induces mucus cell metaplasia in mouse lung. Am. J. Physiol. - Lung Cell. Mol. Physiol. 287, 1293-1302 (2004).

44. Landmann, M. et al. A Semiquantitative Scoring System for Histopathological and Immunohistochemical Assessment of Lesions and Tissue Tropism in Avian Influenza. Viruses 2021, Vol. 13, Page 868 13, 868 (2021).

Acknowledgements: We thank Jim Kellner (Alberta Children's Hospital) and Craig Jenne (University of Calgary) for helpful discussion.

\section{Funding:}

Canadian Institutes of Health Research (CIHR) grant 448323 (DJM) and OV3-172302 (DHE)

\section{Author contributions:}

Conceptualization: DJM, KGP

Methodology: KGP, RSN, CG, CJ, HMT, ED, NF, MMK 
Template revised February 2021

Investigation: KGP, RSN, CG, CMJ, HMT, ED, NF, MMK

Funding acquisition: DJM, DHE

Supervision: DJM, DHE, MMK

Writing - original draft: DJM, KGP

Writing - review \& editing: RSN, DHE

\section{Competing interests:}

RN and DHE hold research contracts and consult privately for Tonix Pharmaceuticals on projects related to Orthopoxvirus-vectored vaccines. The other authors declare that they have no competing interests.

\section{Data and materials availability:}

All data are available in the main text or the supplementary materials. 


\section{Template revised February 2021}

Figure 1: Experimental design. Hamsters were vaccinated with two doses of bnt162b2 delivered intramuscular (IM) and separated by 21 days. Serum neutralizing antibody (NAb) titers were monitored over 8 months by pseudovirus PRNT assay. Two hundred seventy-five days post-first dose, animals were left alone (waning immunity control) or boosted IM with bnt162b2, mRNA-1273, or AZD1222. Recall NAb response was measured on days 3 and 10 post-boost. Fifteen days post-boost (day 290), hamsters were challenged intranasally (IN) with 1e5 plaque forming units (pfu) of the Delta variant of concern (VOC). One day later, the hamsters were cohoused with naïve unvaccinated hamsters for 24 hours in cages that provided ventilated physical separation. Nasal swabs were taken from challenged animals on days 2 and 4 and from cohoused animals on day 2 to assay viral burden. Challenged animals were euthanized on day 4 and lungs harvested to assay viral load and assess lung pathology.

\section{Figure 2: Kinetics of serum neutralizing antibody response after primary and booster} vaccination in hamsters. (A) $\mathrm{NT}_{50}$ against ancestral $\mathrm{SARS}-\mathrm{CoV}-2$ pseudovirus ( $\mathrm{PRNT}_{50}$ assay), measured in serum harvested from hamsters vaccinated with two doses of bnt162b2 separated by vaccination with two doses of bnt162b2, measured using the same assay as in (A). (C) Serum $\mathrm{NT}_{50}$ from animals in (A) plotted by booster group. Booster vaccinations were given on day 275 and serum $\mathrm{NT}_{50}$ measured on days 3 and 10. (D) Serum $\mathrm{NT}_{50}$ in boosted animals (from (C)) plotted from day of boost. Individual data points and/or mean \pm SD is shown. For (A), $n=16$ and these same animals are shown grouped in (C) and (D) (n=4/group). For (B), n=5.

Figure 3: Booster vaccination enhances protection against Delta VOC infection. (A) Animal body weight after Delta VOC challenge plotted as mean percent change relative to day 0. (B) Serum $\mathrm{NT}_{50}$ against authentic Delta VOC (PRNT 50 assay) on day 4 post-challenge. (C-D) 
Template revised February 2021

Infectious Delta VOC titers (plaque assay) in nasal swabs and lung homogenate on day 2 and 4 post-challenge. (E-F) Quantification of Delta VOC nucleocapsid staining in alveoli and interstitium (E) or bronchial epithelium (F) on day 4 post-challenge. (G) Representative images of Delta VOC nucleocapsid staining from (E-F). Red arrows depict alveoli and interstitium staining, while green arrows depict bronchial epithelium staining. Mean \pm SD is shown for linear scales and geometric mean \pm SD for logarithmic scales. $\mathrm{N}=4$ for all groups. Data analyzed by oneway ANOVA followed by Dunnett multiple comparison

\section{Figure 4: Booster vaccination protects against lung pathology and transmission after Delta}

Representative hematoxylin and eosin (H\&E) images of lung inflammation 4 days postchallenge. Red arrows depict airway inflammation while green arrows depict peripheral inflammation. (C) Infectious Delta VOC titers (plaque assay) from nasal swabs taken from naïve hamsters co-housed 1:1 with challenged hamsters. Mean \pm SD is shown for linear scales and geometric mean \pm SD for logarithmic scales. $\mathrm{N}=4$ for all groups. Data analyzed by one-way ANOVA followed by Dunnett multiple comparison test.

\section{Materials and Methods}

\section{Study design:}

Animal experiments and procedures were approved by The University of Calgary Health

Sciences Animal Care Committee (AC20-0053) and the University of Alberta Animal Care and Use Committee (AUP00001847). 6-8-week-old male golden Syrian hamsters (Charles River, Strain Code 049) were used for primary bnt162b2 vaccination and 9-month-old male hamsters 


\section{Template revised February 2021}

were used as age-matched unvaccinated controls for the Delta VOC challenge. Hamsters were vaccinated on day 0 and again on day 21 with a $5 \mu \mathrm{g}$ dose of bnt $162 \mathrm{~b} 2$ by bilateral injection $(2 \times 50 \mu \mathrm{L})$ into the caudal muscle. Blood was collected by retro-orbital bleeding and serum harvested and stored using a standard procedure. Booster vaccination was done with $5 \mu \mathrm{g}$ BNT162b2, $15 \mu \mathrm{g}$ mRNA-1273, or 8e9 viral particles AZD1222 delivered by bilateral caudal muscle injection. All vaccines were acquired from Alberta Health Services (AHS) vaccine clinics after the human doses had been used. Remnant vaccine from each vial was pooled and injected into animals within 6 hours. Fifteen days after booster vaccination (or non-boosted control), animals were challenged in biosafety level 3 with 1 e5 plaque forming unit (pfu) of sequence-verified Delta VOC in a total volume of $100 \mu \mathrm{L}$ delivered intranasal $50 \mu \mathrm{L}$ per nare. Animals were monitored daily by a trained animal technician for signs of clinical illness. Animals were weighed daily, and nasal swabs taken on day 2 and day 4 post-challenge to measure viral burden. Animals were euthanized on day 4 post-challenge, and lungs collected for tissue viral loads and histopathology. To assess transmission, the hamsters were co-housed 1:1 with naïve unvaccinated age-matched male hamsters for 24 hours beginning 24 hours postchallenge. Naïve animals were added to the challenged animal's cage that was modified with a perforated aluminum barrier to physically separate the animals but allow for air exchange. Nasal swabs were taken from co-housed animals on day 2 to measure viral burden. All studies were conducted by blinded experimenters.

For neutralizing antibody measurements in humans, blood was collected from healthy men $(n=3)$ and women $(n=2)$, mean age 44, approximately 14 days after a second dose of bnt162b2 (which was approximately 21 days after their first dose of bnt162b2) under University of Calgary 


\section{Template revised February 2021}

Conjoint Human Research Ethics Board approval REB20-0481 and informed consent. Serum was harvested and stored using a standard protocol.

\section{Cell lines and antibodies:}

Vero (CCL-81) cells were obtained from ATCC and cultured in DMEM (Thermo Fisher

Scientific, Cat. \# 11965118) supplemented with 10\% fetal bovine serum (FBS) (Thermo Fisher

Scientific, Cat. \# 12484028) at $37^{\circ} \mathrm{C}$ in $5 \% \mathrm{CO}_{2}$. Cells were tested and routinely found negative

for mycoplasma by Hoechst 33342 staining (Thermo Fisher Scientific) and fluorescence imaging and LookOut@ Mycoplasma PCR detection kit (Sigma-Aldrich). Primary antibodies used for immunohistochemistry included: Rabbit Anti-SARS-CoV-2 Nucleocapsid (Novus Biologicals, NB 100-56576) and Rabbit Anti-mouse IgG Isotype Control (Novus Biologicals, NBP2-24891). Secondary antibodies used for immunohistochemistry included: EnVision+ Single Reagents, HRP anti-Rabbit (Agilent, K400311-2) and EnVision+ Single Reagents, HRP anti-Rabbit (Agilent, K4001).

\section{Plaque Reduction Neutralization Tests (PRNTs):}

For the pseudovirus PRNT assays, Vero CCL81 cells were seeded into 96-well plates at 20,000 cells/well. Hamster or human serum was heat inactivated for $30 \mathrm{~min}$ at $56^{\circ} \mathrm{C}$, then two-fold serially diluted in PBS. Diluted serum was mixed 1:1 with 100 pfu of replication-competent vesicular stomatitis virus (VSV) pseudotyped with the Spike gene of ancestral SARS-CoV-2 containing a 21 aa $\mathrm{C}$-terminal truncation and expressing green fluorescent protein (VSV- $\Delta \mathrm{G}$ -

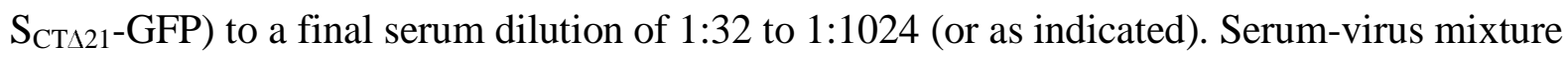
was incubated for 1 hour at $37^{\circ} \mathrm{C}$ then added to Vero cells and incubated for 1 hour at $37^{\circ} \mathrm{C}$. DMEM supplemented with 5\% FBS containing 1\% carboxymethyl cellulose (CMC) was then 
Template revised February 2021

Authentic Delta VOC PRNT assays were done in BSL3 in 12-well plates using serum mixed with authentic Delta VOC (as described above for the pseudovirus assay). Serum-free DMEM media containing $1 \% \mathrm{CMC}$ was added and the cells incubated at $37^{\circ} \mathrm{C}$ for 72 hours followed by fixation and staining with crystal violet. Viral plaques were counted manually from wells containing 15-150 plaques.

For both pseudovirus and authentic virus PRNT assays, the dilution achieving 50\% neutralization $\left(\mathrm{NT}_{50}\right)$ was estimated by nonlinear regression curve fit using GraphPad Prism 9.1.1 (GraphPad Software). All samples were run in duplicate by a blinding technician.

Lungs: The four small right lung lobes were harvested 4 days post-challenge, weighed and homogenized in sterile gentleMACS M Tubes using the gentleMACS tissue dissociator (Miltenyi Biotec) on the EB2.01 program in $2 \mathrm{~mL}$ of DMEM. Tissue homogenates were clarified by centrifugation at 3,000 $\mathrm{x}$ g for $10 \mathrm{~min}$, diluted in serum-free DMEM, and plated in duplicate on Vero cells in 12-well plates. The cells were then cultured in serum-free DMEM containing $1 \% \mathrm{CMC}$ for 72 hours followed by fixation and staining with crystal violet. Viral plaques were counted manually from wells containing 15-150 plaques. 


\section{Template revised February 2021}

Nasal swabs: A sterile polyester swab (Puritan 25-3318-U) was used to swab the nasal cavity, mouth and tongue for approximately 10 seconds. The swab was placed into $500 \mu \mathrm{L}$ of IMDM supplemented with $2 \%$ FCS and stored in screw cap tubes at $-80^{\circ} \mathrm{C}$. For titering virus, nasal swabs were diluted in serum-free DMEM and then processed as described above for lung titers.

\section{Lung histology and immunohistochemistry:}

Four days post-challenge, hamsters were humanely euthanized, and the large right lung dissected and fixed in 10\% neutral buffered formalin (Sigma Aldrich, HT501128) for 48 hours and then embedded into paraffin blocks using a HistoCore Pearl tissue processor (Leica Biosystems). Four $\mu \mathrm{m}$ thick formalin-fixed paraffin embedded (FFPE) sections cut on a Thermo Shandon Finesse 325 microtome (Thermo Fisher Scientific) mounted on SuperFrost plus slides (Thermo Fisher Scientific) were used for hematoxylin and eosin (H\&E) and immunohistochemical (IHC) staining. H\&E was performed using standard procedure. For IHC, slides were deparaffinized in xylene and rehydrated through a series of graded ethanol to distilled water. Heat-induced epitope retrieval was performed using a pressure cooker on steam setting. Slides were placed in sodium citrate buffer (10 mM sodium citrate, $0.1 \%$ Tween-20, $\mathrm{pH} \mathrm{6.0)}$ and boiled for $20 \mathrm{~min}$ at $65^{\circ} \mathrm{C}$, treated with $3 \%$ hydrogen peroxide, rinsed in phosphate-buffered saline with $0.1 \%$ Tween-20 (PBST) and then protein blocked (Avidin Biotin Blocking Kit, ab64212). Slides were incubated in primary antibody diluted in $1 \%$ BSA for 1 hour at room temperature (RT) then washed twice for three minutes in Tris-buffered saline with 0.1\% Tween-20 (TBST). Envision+ HRP-labeled secondary antibody was added to slide and incubated $30 \mathrm{~min}$ at RT and washed twice for three minutes with TBST. Slides were incubated with DAB (Agilent, GV82511-2), washed twice for three minutes with TBST, then rinsed in running tap water. 


\section{Histology scoring for inflammation:}

H\&E-stained slides were evaluated by a blinded pulmonary pathologist using a light microscope. A semi-quantitative histological scoring system ${ }^{41-43}$ was adapted for scoring inflammation. Each space was scored independently and the final score for each animal was calculated as the sum of all scores.

\begin{tabular}{|c|c|c|}
\hline Score & $\begin{array}{c}\text { Bronchovascular (BV) bundle } \\
\text { inflammation }\end{array}$ & $\begin{array}{c}\text { Alveolar and Interstitial } \\
\text { inflammation }\end{array}$ \\
\hline 0 & Nil & Nil \\
\hline 1 & $\begin{array}{l}0-5 \% \text { of BV bundles cuffed by inflammatory } \\
\text { cells, generally scattered small groups of } \\
\text { cells, < } 5 \text { cells thick }\end{array}$ & $\begin{array}{l}\text { A few macrophages only present in } \\
\text { the alveolar spaces with minimal } \\
\text { adjacent interstitial inflammation (0- } \\
5 \% \text { of the lung area) }\end{array}$ \\
\hline 2 & $\begin{array}{l}>5-30 \% \text { of BV bundles cuffed by } \\
\text { inflammatory cells, generally scattered small } \\
\text { groups of cells, }<5 \text { cells thick but some }>5 \\
\text { cells thick }\end{array}$ & $\begin{array}{l}>5-30 \% \text { of alveoli and interstitium } \\
\text { inflamed }\end{array}$ \\
\hline 3 & $\begin{array}{l}>30-60 \% \text { of BV bundles cuffed by } \\
\text { inflammatory cells, generally scattered small } \\
\text { groups of cells, < } 5 \text { cells thick but some }>5 \\
\text { cells thick }\end{array}$ & $\begin{array}{l}>30-60 \% \text { of alveoli and interstitium } \\
\text { inflamed }\end{array}$ \\
\hline
\end{tabular}




\begin{tabular}{|c|l|l|}
\hline 4 & $>60 \%$ of BV bundles cuffed by inflammatory & $>60 \%$ of alveoli and interstitium \\
& cells, generally $>5$ cells thick & inflamed
\end{tabular}

\section{Histology scoring for SARS-CoV-2 nucleocapsid staining:}

Nucleocapsid-stained slides were evaluated by a blinded pulmonary pathologist using a light microscope. A semi-quantitative histological scoring system ${ }^{44}$ was adapted for scoring the viral load as follows:

1. Airway bronchial epithelial cells (BEC) staining positively for SARS-CoV-2 nucleocapsid antigen were expressed as a percentage of total BECs: $0=$ no positive cells, $1=$ scanty $(<10 \%$ of BECs or only positive necrotic cells in the bronchial lumen $), 2=10$ $30 \%$ BECs, 3 = >30-60\% BECs, $4=>60-80 \%$ BECs, $5=>80 \%$ BECs.

2. SARS-CoV-2 nucleocapsid antigen expression in the lung periphery (alveoli) was assessed by counting positive cells in the lung section, excluding the bronchial epithelium, using the $20 \times$ objective (200x power). The scores for each lung section were expressed as the number of positive cells per $\mathrm{cm}^{2}$ lung area.

Each space was scored independently and the final score for each animal was the sum of the scores. All scores were normalized to naïve animals.

\section{Statistical analyses:}

Data analysis was performed using GraphPad Prism 9.1.1 (GraphPad Software). Statistical tests, number of animals (or samples), average values, and statistical comparison groups are indicated in the figures and legends. All values are reported as mean or geometric mean $\pm \mathrm{SD}$. 
bioRxiv preprint doi: https://doi.org/10.1101/2021.12.27.474282; this version posted December 28,2021 . The copyright holder for this preprint (which was not certified by peer review) is the author/funder, who has granted bioRxiv a license to display the preprint in perpetuity. It is made availablestidiniafeQ

Template revised February 2021 
bioRxiv preprint doi: https://doi.org/10.1101/2021.12.27.474282; this version posted December 28, 2021. The copyright holder for this preprint (which was not certified by peer review) is the author/funder, who has granted bioRxiv a license to display the preprint in perpetuity. It is made available under aCC-BY-NC-ND 4.0 International license.

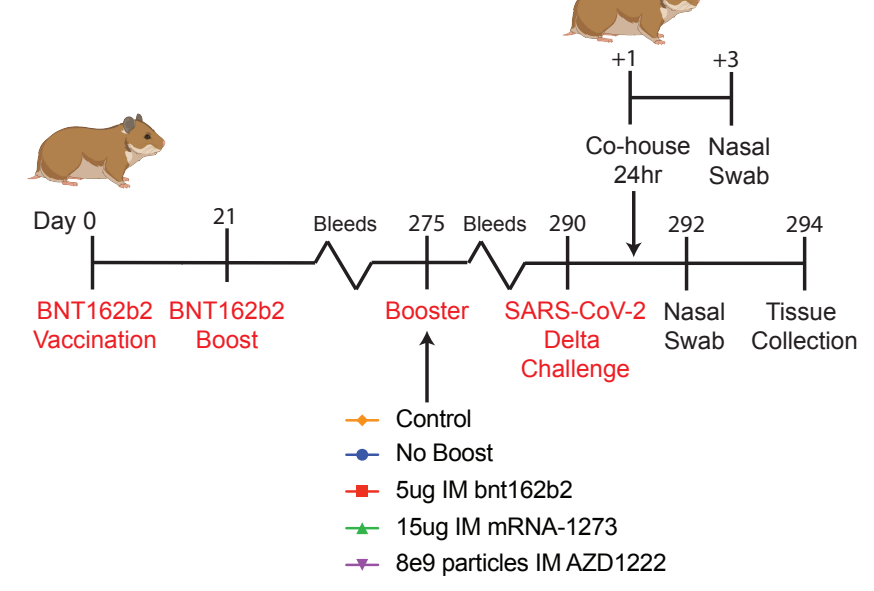


bioRxiv preprint doi: https://doi.org/10.1101/2021.12.27.474282; this version posted December 28, 2021. The copyright holder for this preprint (which was not certified by peer review) is the author/funder, who has granted bioRxiv a license to display the preprint in perpetuity. It is made A
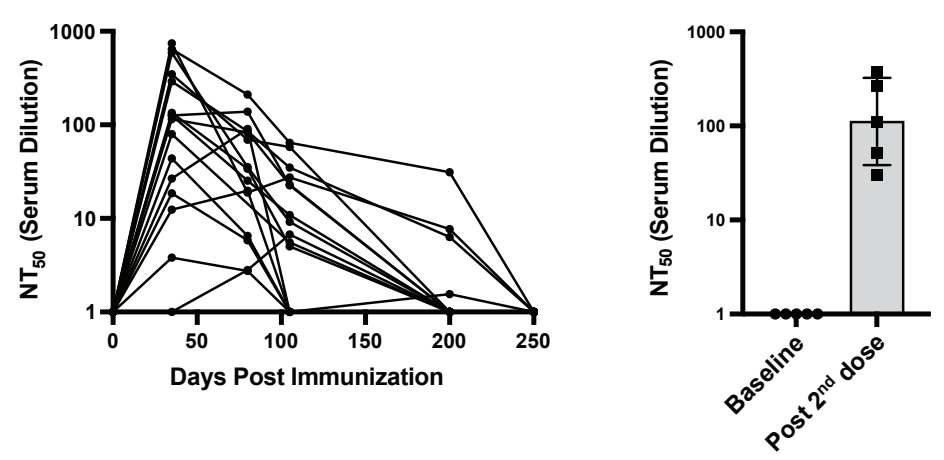

C

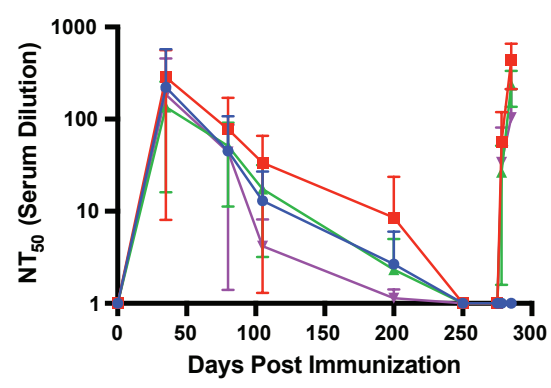

D

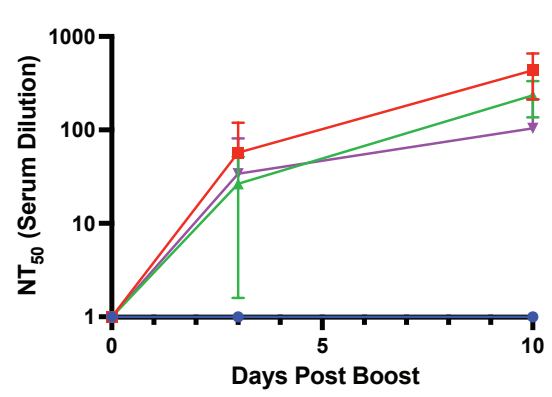

$\rightarrow$ Control

- No Boost

- 5 ug IM bnt162b2

- 15ug IM mRNA-1273

$\rightarrow \quad 8$ e9 particles IM AZD1222 
bioRxiv preprint doi: https://doi.org/10.1101/2021.12.27.474282; this version posted December 28, 2021. The copyright holder for this preprint (which was not certified by peer review) is the author/funder, who has granted bioRxiv a license to display the preprint in perpetuity. It is made

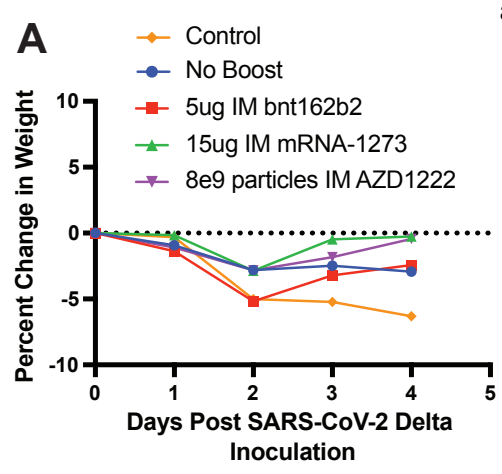

E

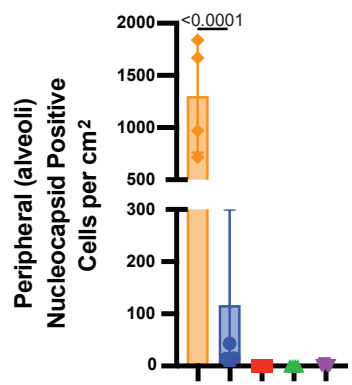

F
B

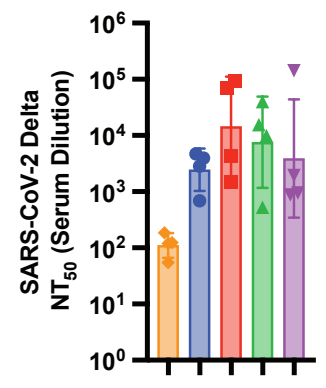

G
C

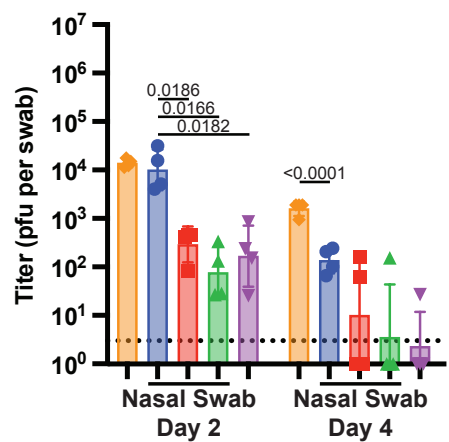

D

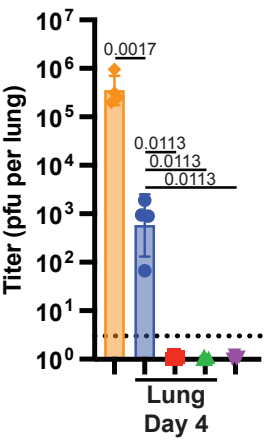

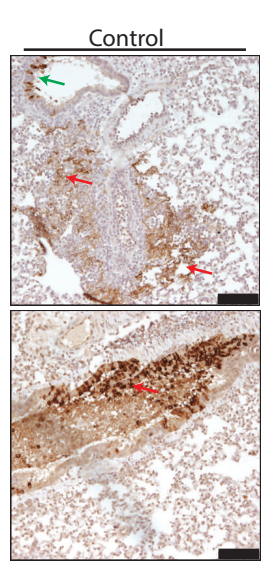

Representative
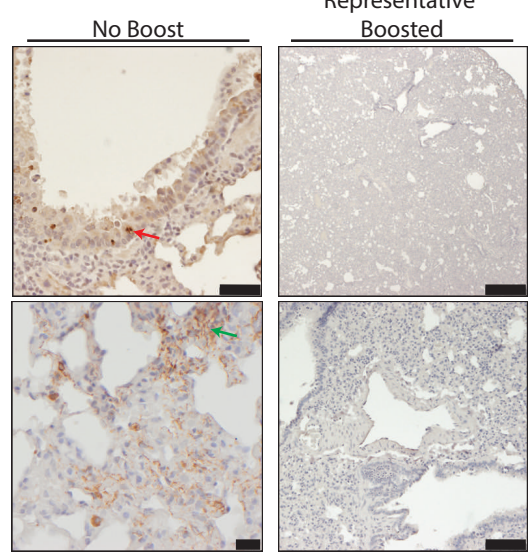
bioRxiv preprint doi: https://doi.org/10.1101/2021.12.27.474282; this version posted December 28,2021 . The copyright holder for this preprint
A (which was not detified by peer review) is the author/funder, who has granted bioRxiv a license to display the preprint in perpeCQity. It is made
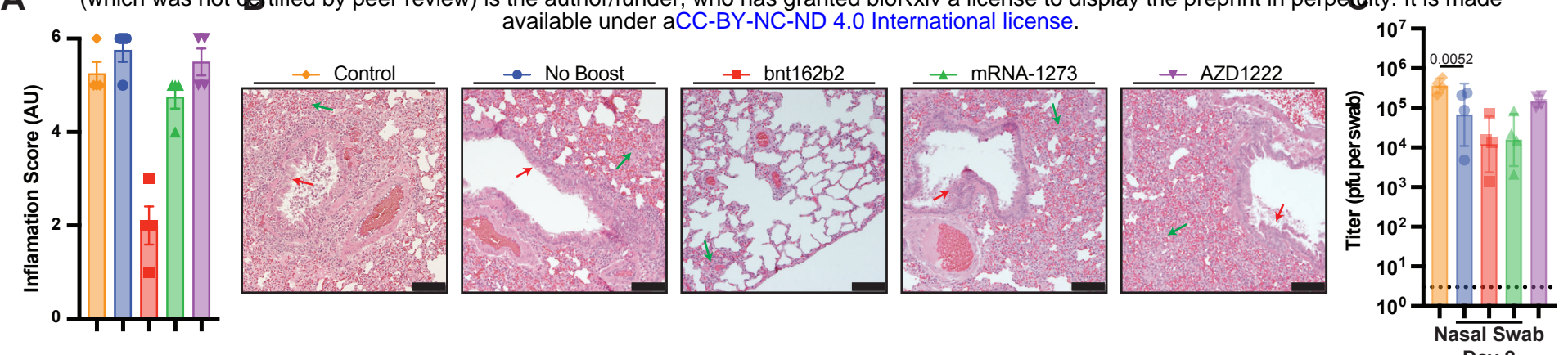